\title{
Corela
}

Cognition, représentation, langage

HS-22 | 2017

Prédication et prépositions en anglais

\section{La prédication : approche de la théorie des opérations prédicatives et énonciatives}

Jean-Marie MERLE

\section{(2) OpenEdition}

Journals

Édition électronique

URL : http://journals.openedition.org/corela/4959

DOI : 10.4000/corela.4959

ISSN : 1638-573X

Éditeur

Cercle linguistique du Centre et de l'Ouest - CerLICO

Référence électronique

Jean-Marie MERLE, «La prédication : approche de la théorie des opérations prédicatives et énonciatives », Corela [En ligne], HS-22 | 2017, mis en ligne le 05 novembre 2017, consulté le 19 avril 2019. URL : http://journals.openedition.org/corela/4959; DOI : 10.4000/corela.4959

Ce document a été généré automatiquement le 19 avril 2019

\section{(c) (i) (2)(2)}

Corela - cognition, représentation, langage est mis à disposition selon les termes de la licence Creative Commons Attribution - Pas d'Utilisation Commerciale - Partage dans les Mêmes Conditions 4.0 International. 


\title{
La prédication : approche de la théorie des opérations prédicatives et énonciatives
}

\author{
Jean-Marie MERLE
}

\section{Introduction}

1 Afin de traiter de la prédication en prenant comme support d'analyse le texte d'Ann Tyler extrait de The Accidental Tourist, on commencera par définir la notion de prédication comme opération énonciative structurante $(\S 1)$ - et on examinera trois approches différentes de ce phénomène - approche néoplatonicienne, approche néoaristotélicienne, approche calquée sur la structure informative - ainsi que les avantages et les inconvénients de chacune. La prédication est une opération énonciative structurante à laquelle sont associées les modalités énonciatives qui caractérisent l'énoncé, et on envisagera successivement, en relation avec la prédication, la question de la modalité énonciative et celle de la plurimodalité (\$2). On observera ensuite (\$3) deux phénomènes connexes de la prédication: la biprédication, dans un contexte de coordination; et la coprédication, représentée par l'apposition, l'attribut de l'objet, les nexus résultatifs. On s'interrogera sur les subordonnées relatives intégrées ou sur les subordonnées nominales (enchâssées), et sur les raisons pour lesquelles elles ne donnent pas lieu à prédication $(\S 4)$. On envisagera le lien entre prédication et circonstants ( $(5)$, puis un cas particulier de prédication ( $\$ 6)$, la prédication sans relation prédicative. Les deux étapes suivantes comporteront l'amorce de deux typologies: celle du lien entre prédication et types de discours ( $(7)$; puis celle des types de prédication ( $(8)$. La dernière partie $(\$ 9)$ sera consacrée à cinq études de cas, dont la dernière sera l'occasion de revenir sur le carré des modaux d'Adamczewzki et Gabilan (1993). 


\section{La prédication - essai de définition - 3 approches différentes}

\subsection{Définition de la prédication}

2 La notion de prédication présente un intérêt si elle ne fait pas double emploi avec celle de relation prédicative. Elle a un pouvoir explicatif considérable, dans la mesure où elle renvoie à l'une des opérations qui structurent l'énoncé.

Définition - La prédication, en linguistique, peut se définir sommairement comme l'opération par laquelle se structure la relation fondamentale - celle qui canoniquement fonde l'énoncé - et, par transfert métonymique, comme le produit de cette opération. Autrement dit, il s'agit de l'assemblage prédicatif auquel sont associées les modalités énonciatives qui caractérisent l'énoncé (cf. Merle, 2009a : 51).

Étant donné le rôle fondamental de cette opération dans la structuration des énoncés, la notion de prédication occupe une place non négligeable dans nombre d'appareils descriptifs, notamment dans la description des cadres prédicatifs, des structures actancielles, des phénomènes co-prédicatifs (appositions ou adjoints prédicatifs).

5 Trois approches de la prédication

6 Trois approches de la prédication se dégagent de la littérature contemporaine sur la question : l'une néoplatonicienne, la seconde néo-aristotélicienne, la troisième calquant la notion de prédication sur la structure informative.

\subsection{Les origines de la notion de prédication}

7 L'intuition linguistique la plus ancienne à laquelle nous ayons accès, et qui inspire la tradition occidentale, remonte au IVe siècle av. J.-C. Déjà on peut percevoir deux conceptions différentes, de l'énoncé, d'une part, et de l'assemblage prédicatif, d'autre part - chez Platon, puis chez les Stoïciens, d'une part, et chez Aristote, d'autre part - deux conceptions qui n'entrent pas pour autant en contradiction.

8 F. Ildefonse (1994:3) montre que

la problématique de la prédication est bel et bien ouverte par Platon, dont l'enjeu fondamental [...] paraît être plus précisément [...] la fondation de l'énoncé, ce par la théorie du mélange des genres.

9 À la fin du Sophiste (261e-262e), dans sa célèbre leçon de grammaire, Platon inaugure la forme canonique de l'expression, le prôtos logos :

[...] les paroles proférées ne font référence ni à une action, ni à une absence d'action, ni à la réalité d'un être ou d'un non-être, tant qu'on n'a pas mêlé les noms et les verbes: alors seulement, il $\mathrm{y}$ a ajustement et dès lors cette combinaison élémentaire devient énoncé, pratiquement le premier et le plus petit des énoncés.... C'est pourquoi, dans ce cas, nous parlons d'énoncer et non pas seulement de nommer et c'est précisément à cet agencement que nous donnons le nom d'énoncé. ${ }^{2}$.

10 Le principe de la prédication est ainsi le

dire d'une chose non seulement sa désignation spécifique, mais beaucoup d'autres choses différentes ${ }^{3}$

11 Autrement dit, la prédication ouvre la possibilité de produire une infinité d'énoncés. 

kategorema, que nous percevons comme l'ancêtre du couple sujet-prédicat -, Aristote en arrive à définir l'assemblage substrat-états comme une relation d'interdépendance :

... aucun [état] n'a par lui-même naturellement une existence propre, ni ne peut être séparé de la Substance $[. . .]^{5}$

\subsection{Trois appréhensions différentes}

De ces deux conceptions sont issues des appréhensions différentes d'un même phénomène. On peut en identifier trois qui coexistent aujourd'hui, l'une, centrée sur le prédicat ; une seconde qui garde en mémoire la préexistence du substrat ; une troisième qui assimile la prédication à la structure thématique.

\subsubsection{Un prédicat central}

La première se trouve chez les Stoïciens et dans la définition que donne Diogène Laërce ${ }^{6}$ du prédicat (tò kategoréma) : «[...] un dit incomplet qui, construit avec un cas direct, engendre une proposition ». Les Stoïciens définissent le prédicat et l'assemblage prédicatif, mais on ne trouve pas chez eux le couple sujet-prédicat. On peut y voir une parenté entre la conception stoïcienne de la prédication et la conception platonicienne. Les Stoïciens, en plaçant le prédicat au centre de la prédication, sont amenés à élaborer une classification des prédicats, selon la personne, la valence et la diathèse, le verbe étant envisagé comme un prédicat non composé. Cette conception se trouve abondamment reprise et développée au $\mathrm{XX}^{\mathrm{e}}$ siècle, dans la Syntaxe structurale de Tesnière, par exemple, puis dans tous les travaux faisant depuis les dernières décennies du XXe siècle une place aux interactions entre sémantique lexicale et constructions, donnant une prépondérance au prédicat, et notamment au prédicat verbal, selon une approche que l'on nommera pour cette raison néoplatonicienne.

\subsubsection{Place centrale du prédicat dans la théorie des opérations énonciatives}

La relation fondamentale, dans la Théorie des Opérations Énonciatives (TOE), se représentera schématiquement comme une relation structurée autour d'un élément central $\mathrm{r}$ (représentation $1: \mathrm{arb}$ ), cet élément relateur et cohéreur étant canoniquement un verbe (pumped dans l'exemple 2).

1. arb

2. (1. 69) Macon pumped his brakes...

17 Pump a un fonctionnement de verbe à deux places - c'est-à-dire de verbe transitif -, qui laisse attendre deux arguments dans sa structure, ou deux actants dans son schéma actanciel (sa structure sémantique). Autrement dit, pumped est l'élément central autour duquel se construit la prédication, par instanciation des places d'arguments, par Macon et par his brakes. Les notions employées dans la TOE concernant la structuration d'un énoncé sont les suivantes :

18 - schéma de lexis (trois lexèmes ${ }^{7}$ : Macon, brakes, pump);

Corela, HS-22 | 2017 
19 - relation primitive (sémantique) : pump implique un agent (Macon) et à son dynamisme correspond une orientation de l'agent vers le patient (brakes), qui transite précisément par le verbe (pump) ;

20 - relation prédicative (structurée) : choix d'un «terme de départ », Macon, agent, ce qui entraîne une diathèse active ; représentation de la relation prédicative :<Macon-pump his brakes>.

21 Sur ce point, on pourra comparer avec un schéma rencontré à la ligne 33 - it was uttered -, dans lequel le "terme de départ ", it, référant à gasp of relief, entraîne une diathèse passive dans la subordonnée temporelle before it was uttered.

22 - relation énonciative : par rapport à une situation d'énonciation Sit 0 , la détermination temps-aspect-modalité est apportée à la relation prédicative qui accède au statut d'énoncé. Dans Macon pumped his brakes, le temps est le prétérit, marqueur d'une opération de rupture entre Sit1 (situation d'énoncé) et Sit0 (situation d'énonciation). Ici, l'aspect zéro est la marque, dans l'énoncé, d'un point de vue global. La référence construite par cet énoncé est référence à un événement borné à gauche et à droite (approche topologique caractéristique de la TOE), qui entre dans la succession des événements qui constituent le récit, le prétérit étant prétérit aoristique. Sur le plan modal, il s'agit d'une assertion.

\subsubsection{Sujet ou « terme de départ »}

La TOE nomme "terme de départ " le sujet d'un énoncé, en raison de sa fonction structurante, liée comme on vient de le voir à son sémantisme, qui, de la relation primitive (sémantique) à la relation prédicative (structurée), détermine la diathèse de l'énoncé. Ainsi l'énoncé de l'exemple 3 a beau être interrogatif, you est identifiable comme «terme de départ » en raison de ses propriétés structurantes, même s'il n'occupe plus en l'occurrence la position initiale par défaut propre au sujet en contexte noninterrogatif.

3. (1.36) Did you notice that boy with the motorcycle?

24 Le «terme de départ », ou argument sujet, a des propriétés qui le distinguent des autres arguments. Voici quelques-unes des propriétés subjectales :

- statut nominal du sujet, qu'on ait affaire à un syntagme nominal à noyau lexical, comme the sky (1. 9), dans the sky grew almost black, ou à un pronom ${ }^{8}$, comme he (1. 8), dans He'd kept away from the sun ;

26 - position initiale : à la l.1, They occupe la position initiale dans They were supposed to stay ; mais comme on l'a vu plus haut, la notion de «terme de départ » n'est pas liée à la position initiale, mais à la fonction structurante du sujet ;

- nominatif : le nominatif (cas du nom dans sa fonction de base de dénomination) est le cas du sujet lorsqu'il est porteur de marque casuelle ; ainsi they, à la l. 1, est par défaut un nominatif en sa qualité de sujet;

27 - inversion auxiliaire-sujet dans les structures interrogatives directes. You (dans l'exemple 3, 1. 36), quitte sa position initiale tout en ayant la fonction structurante de sujet: le schéma de l'inversion s'interprète comme une mise en question de la structure prédicative ;

- reprise dans un tag; ainsi you (1. 48), dans le tag 'Do you', reprend you sujet de you [don't] care that much. 
- accord; l'accord est un phénomène sémantico-référentiel. On observe l'accord, par exemple, entre they (référent pluriel) et were, dans They were supposed to stay (l. 1), ou encore entre I (référent singulier identifié à la source énonciative) et am dans I'm trying to be (1.62).

Autrement dit, les propriétés subjectales, dans les langues où elles sont identifiables dont font partie l'anglais et le français -, donnent un statut à part à l'argument sujet, souvent décrit alors comme "argument externe", ce qui permet de ré-envisager la prédication sous un angle différent.

\subsubsection{Une relation apport-substrat}

Une autre conception de la prédication garde en mémoire le statut à part du sujet, notamment sur le plan sémantico-référentiel. Cette conception, qui prend en compte l'existence, ou la préexistence, du substrat, et qui est héritée du couple aristotélicien hupokeimenon-kategorema (sujet-prédicat), ouvre au Moyen Âge sur une distinction tranchée entre logique et grammaire. À la fin du XIII ${ }^{e}$ siècle, Jean de Dacie et Martin de Dacie laissent le couple sujet-prédicat aux logiciens et ont recours, en grammaire, au couple suppositum-appositum (suppôt-appôt ou support-apport). De ce couple restera dans la terminologie le terme d'apposition, qui, après avoir décrit au Moyen Âge ce que nous appelons maintenant de nouveau prédicat, servira à décrire un coprédicat (ajout prédicatif ou angl. predicative adjunct), autrement dit soit un dédoublement coprédicatif, soit une greffe coprédicative ${ }^{9}$.

De cette conception restera aussi l'appréhension de la relation sujet-prédicat comme une relation entre un apport et un élément stable, autrement dit comme un apport sémantico-référentiel sur un support référentiel et structurel.

Deux approches différentes se dessinent donc: l'une, néoplatonicienne, qui place le prédicat au centre de l'énoncé. L'autre, néo-aristotélicienne, qui privilégie une conception binaire de la relation prédicative (structuration apport-support).

\subsubsection{Troisième approche : la prédication vue comme structure informative}

Une troisième conception, enfin, tend à faire coïncider la prédication avec la structure thématique, le sujet étant alors défini comme "ce dont on parle " (le thème) et le prédicat comme « ce qu'on en dit » (le rhème). La prédication n'est plus envisagée comme la structure modalisée qui fonde l'énoncé, mais comme un agencement informatif canonique. Cette définition ne tient pas compte de la possibilité de dissocier le thème du sujet offerte par les constantes structurelles d'une langue donnée ${ }^{10}$.

La problématique ouverte par les énoncés thétiques (qui inaugurent un thème nouveau), dans lesquels aucun élément n'a de statut thématique, est par la force des choses l'arrêt de mort de la prédication, dans la mesure où l'absence de thème ne permet pas de retrouver de structure thème-rhème. Cette approche, selon le point de vue adopté ici, n'a pas recours à la notion de prédication pour décrire les constantes de la structuration des énoncés : les énoncés thétiques ne sont pas considérés comme des prédications. Dans le texte d'Ann Tyler, on relève les énoncés thétiques suivants (4 à 7), dont le sujet ( $a$ wide lake, a mobile home, a gust of wind, great lashings of water) n'a pas de statut thématique et se caractérise par sa faible détermination.

4. (1. 68) A wide lake [...] in the center of the highway crashed against the underside of the car [...] 
5. (1. 78) 'A mobile home's washed clear across that trailer park.'

6. (1.16) Every now and then a gust of wind blew up.

7. (1. 46) Great lashings of water flung themselves at the windshield.

l'état de fait ou sur l'événement dans la représentation desquels il joue un rôle sémantique ; (dans Macon pumped his brakes, Macon préexiste à l'événement représenté par Macon pumped his brakes). Si l'on ré-envisage les énoncés thétiques de la série 4 à 7 (cf. § 1.3.5), on voit que le référent de a mobile home, 1. 78, préexiste à l'état de fait énoncé ('A mobile home's washed clear across that trailer park'). Il en est de même de a wide lake, 1. 68. En revanche, a gust of wind (ex. 6, 1. 16) et great lashings of water (ex. 7, 1.46) n'acquièrent d'existence que dans le cadre de la référence à l'événement construite autour des procès blew up (1. 6) et flung themselves at the windshield (1.46). Par ailleurs, un sujet n'a pas toujours de valeur référentielle (cf. it, 1. 40). L'antériorité référentielle du sujet n'est donc qu'une tendance.

40 2) le fait que l'assemblage sujet-prédicat corresponde à une grammaticalisation du couple thème-rhème, ce qui affranchit le sujet de son statut originel de thème (cf. Albrespit (2003), ou Lazard (1998, 2003a et 2003d), et cf. § 1.3.5)...

41 Voici un exemple (pris en dehors du texte) de thème coïncidant avec le Complément de rang 1 (c1), régime direct de would have stressed:

Two details only bear importantly upon the story : his intense bitterness against the swindling guardian, whom he knew personally, and the question of what he could do to earn his living. These two, had he written the truth for the symposium, Mr. Adam would have stressed. (Algernon Blackwood, 1949) 
She laid the stick and her baby on the grass while she untied the rope that tethered the calf. The length of the rope separated them. The cow was near the calf, and both were lying down. Feed along the creek was plentiful, and every day she found a fresh place to tether it, since tether it she must, for if she did not, it would stray with the cow out on the plain. (Barbara Banyon) permet à un sujet impersonnel et non référentiel de recevoir les propriétés du sujet syntaxique (accord, inversion, reprise) ; dans l'exemple 8 (1. 40),

8. (1. 40) It's crazy to ride a motorcycle on a day like today. it reçoit bien les propriétés subjectales d'un sujet syntaxique :

$1 /$ accord réalisé (sur la base des propriétés sémantiques de it, qui en tant que pronom neutre, n'est ni masculin, ni féminin, ni pluriel) : it + sg de $3^{\mathrm{e}}$ personne is ;

2/ manipulation-inversion : is it [so] crazy / isn't it crazy to ride a motorcycle on a day like today?

3/ manipulation-reprise : it's crazy to ride a motorcycle on a day like today, isn't it ?

4/ l'accord et l'inversion, que l'on peut prendre respectivement et solidairement comme indice de l'incidence (et de la relation sémantico-référentielle) fondamentale et comme indice du rôle de l'incidence fondamentale dans la structuration de l'énoncé, jusque dans son statut illocutoire (modalité énonciative de base) ;

5/ la possibilité de construire un assemblage prédicatif, et un énoncé (syntagme ou nexus + intonation + indexation; cf. Merle, 2006a, 2006b, 2008), sans recourir à un verbe.

Si le premier facteur n'est qu'une tendance, les quatre derniers sont de poids: la description de l'assemblage syntaxique qui a le plus grand pouvoir généralisant est peutêtre en définitive celle qui donne le prédicat comme apport et le sujet comme support. C'est pour cette raison aussi que la notion de relation d'incidence s'applique à la description de la structure syntaxique. Les relations d'incidence mettent en place les relations structurelles. La structure qui se construit autour du verbe, quant à elle, génère un schéma actanciel (ou structure sémantique).

Si on définit la prédication comme opération structurante, et comme le produit de cette opération muni de modalité énonciative, on aura par défaut une relation nouée autour d'un verbe, la fonction verbale étant nodale, modale, prédicative. Il s'agit de la structuration prototypique et du cas le plus abondamment représenté dans le texte.

\section{Prédication et modalité}

La question de la modalité est cruciale. Elle est souvent traitée soit comme le statut illocutoire d'un énoncé, soit comme le paradigme des opérateurs et des modaux qui se trouve au cœur du système verbal de l'anglais. Dans les faits, la modalité énonciative est composite.

\subsection{Statut illocutoire et modalité énonciative}

La classification de la modalité énonciative dans la TOE correspond de façon assez étroite au statut illocutoire des énoncés. 


\subsubsection{Type déclaratif}

\subsubsection{Type interrogatif}

10. (1. 81) 'What did I say?'

9. (1. 62) 'I'm trying to be.'

Au type déclaratif correspond la modalité assertive.

Le type interrogatif correspond à la modalité épistémique (énoncé d'un problème de connaissance).

\subsubsection{Type exclamatif}

11. (1. 74) 'You and your systems!'

À l'exclamation correspond une surcharge d'expressivité, et une affinité avec la modalité appréciative. L'énoncé 11 de la 1.74 se construit sur une apostrophe et sur une coordination à fonction appréciative (péjorative).

\subsubsection{Type injonctif}

12. (1. 78) 'Will you look at that !'

$\mathrm{Au}$ type injonctif correspond la modalité radicale déontique. On pourrait avoir : Look at that ! (rb imposé au destinataire de l'énoncé), qui serait du même ordre que : 'Listen.' (1. 84) Un corollaire de cette remarque sur le fait que la modalité énonciative correspond au statut illocutoire, est qu'il existe par défaut une prédication par énoncé, même dans les cas de subordination. On reviendra sur ce point.

\subsection{Prédication et plurimodalité}

Dans les faits, la modalité est le plus souvent composite. En voici quelques preuves.

8. (1. 40) 'It's crazy to ride a motorcycle on a day like today,'

13. (l. 42) 'We could do that,'

14. (1. 25) 'Maybe you should put on your glasses.'

15. (1. 82) 'I just can't live with you anymore,'

Dans l'exemple 8, on a affaire à l'assertion d'une modalité appréciative, it's crazy, portant sur le contenu propositionnel extraposé to ride a motorcycle on a day like today.

Dans l'exemple 13, la suggestion we could do that se construit sur l'assertion d'un possible (radical dynamique).

Dans l'exemple 14, l'énoncé sarcastique maybe you should put on your glasses comporte une modalité radicale déontique (should) modifiée par une modalité épistémique (maybe).

Dans l'exemple 15, l'énoncé déclaratif I just can't live with you anymore se construit sur l'assertion de la négation d'un possible (radical dynamique). 


\section{Biprédication et coprédication} r"b") $\left.[\mathrm{b}],\left[\mathrm{b}^{\prime}\right]\right)$ [coord] a'r'b'. passed).

La biprédication se caractérise par la coexistence de deux prédications, chacune étant susceptible de comporter sa propre modalité. C'est un phénomène ordonné, qui correspond aux cas de coordination (syndétique ou asyndétique).

La coprédication, quant à elle, se caractérise par sa dépendance, par rapport à la prédication. La dépendance coprédicative est représentée par l'apposition, l'attribut de l'objet, les nexus résultatifs.

\subsection{Coordination et biprédication}

La coordination de relations prédicatives donne lieu à prédication multiple :

16. (1. 9-10) [...] the sky grew almost black and several enormous drops spattered the windshield. (arb [coord] a'r'b')

17. (1.1-2) They were supposed to stay at the beach a week, but neither of them had the heart for it, and they decided to come back early. (arb [coord] a'r'b' [coord] a"

18. (1. 69) Macon pumped his brakes and drove on. (a[rb coord $\left.\mathrm{r}^{\prime} \mathrm{b}^{\prime}\right]$ )

19. (1. 13-14) They passed a pickup truck, then a van all covered with stickers [...] (ar

L'exemple 16 (1. 9-10) est un cas de biprédication, qui comporte deux assertions (the sky grew almost black et several enormous drops spattered the windshield), selon le modèle arb

L'exemple 17 (1. 1-2) comporte trois prédications (1/ They were supposed to stay at the beach a week; 2/ neither of them had the heart for it ; 3 / they decided to come back early), chacune relevant de la modalité assertive. Ces trois prédications sont ordonnées : la troisième est assertée comme suite logique de la deuxième ( $\mathrm{cf}$. and $=>s o=>$ and consequently), et la deuxième est assertée en opposition à la première (cf. but).

L'exemple 18 (1.69) est également un cas de biprédication. On a affaire à une coordination de deux prédicats, pumped his brakes et drove on, incidents à un même sujet, selon le schéma a[rb coord r'b']. Chaque relation est assertée.

Dans l'exemple 19, il n'y a pas de coordination au sens étroit du terme, mais, au sens large, coordination zéro, ou asyndétique, qui construit la représentation de deux événements successifs, en contexte aoristique, par la mise en relation d'un complément ( $a$ pickup truck) puis d'un autre (a van) avec le même sujet (they) via le même élément verbal (

69 On ne trouve pas dans le texte d'exemple de biprédication comportant deux modalités énonciatives différentes, mais une manipulation est parfaitement possible, qui donne un statut énonciatif complet dans les deux cas à chacun des segments coordonnés en lui associant une modalité différente :

18'. (1. 69) Macon pumped his brakes, [but did he drive on ?]

20. (1. 90) You never did like moving[, or did you ?] 


\subsection{Apposition et coprédication}

70 Le phénomène de coprédication implique, comme dans l'exemple 21, une dépendance étroite entre un coprédicat à mode non fini (le syntagme participial leaning her head against the side window) et une structure principale (Sarah sat next to him), qui fournit un support (ici, Sarah), point de contact grâce auquel s'opère l'indexation énonciative du coprédicat (dépendant) sur la prédication (centrale) :

21. (1. 2) Sarah sat next to him, leaning her head against the side window.( arb, $\underline{3}$

$\left.\mathrm{r}^{\prime} \mathrm{b}^{\prime}\right)$ ( $\underline{3}$ : opérateur de repérage)

71 Le syntagme participial est apposé, ce qui correspond à une double relation d'incidence, celle du prédicat sat next to him, incident à Sarah, et celle du coprédicat participial leaning her head against the side window, incident également à Sarah. Dans la TOE, Sarah est terme de départ, et support, de chacune des deux relations prédicatives.

Remarque : l'apposition se distingue très nettement du circonstant par sa fonction.

73 1) Le circonstant est modifieur d'une relation prédicative, comme dans l'exemple 22 (1.58), qui contient deux nexus circonstants (1/ their windows opaque; 2/ their gleaming surfaces bouncing back the rain), circonstants modifieurs de that had parked at the side of the road:

22. (1. 58) [...] that had parked at the side of the road, their windows opaque, their gleaming surfaces bouncing back the rain.

2) Dans l'exemple 21 (1.2), ce n'est pas Sarah sat next to him qui est concerné par leaning her head against the side window, mais Sarah. A la différence du circonstant, qui est incident à une relation d'incidence, l'apposition est incidente à un support nominal.

3/ La relation étroite entre prédication et coprédication, en revanche, peut se gloser à l'aide de circonstants : l'exemple 21 peut se gloser as Sarah sat next to him, she leant her head against the side window, ce qui ne fait pas pour autant ${ }^{12}$ de Sarah sat next to him un circonstant de la relation coprédicative dans l'exemple 21.

La fonction coprédicative caractéristique de l'apposition est facilement occultée, alors qu'elle mérite considération.

En latin, Gaffiot traduit appositio par « action d'appliquer, application, action d'ajouter, ajout ». Boèce, au début du VIe siècle, utilise déjà la notion d'adpositio qu'il paraphrase à l'aide de la notion de praedicatio :

Adpositiones id est praedicationes (Boèce, cité par Rosier, 1994 : 98) ${ }^{13}$

L'emploi de la notion d'apposition se généralise à la fin du XIIe siècle, dans le couple suppositum-appositum, qui est donc à l'origine un des avatars du couple sujet-prédicat, du couple hupokeimenon-categorema employé par Aristote, et du couple subjectum-praedicatum , que les grammairiens du Moyen Âge laissent aux logiciens, comme le montre la citation de Jean de Dacie :

Quicquid est in oratione perfecte constructa, vel est subjectum, seu suppositum grammatice loquendo, vel est praedicatum, seu appositum, vel est dispositio alterius istorum vel utriusque. (Jean de Dacie, Summa grammatica, ca. 1280)

Tout ce qui est dans un énoncé construit de manière parfaite, soit est un sujet, ou un suppôt, pour parler en grammairien, soit est un prédicat ou un appôt, soit est ce qui est une détermination de l'un ou de l'autre. (trad. Rosier, 1994 : 81) 

un cadre beaucoup plus vaste, sous les espèces du couple systématisé par Guillaume, d'apport et de support, qui décrit le phénomène d'incidence ; 2/ dans la notion d'apposition, qui est une forme particulière d'apport, apport d'ordre co-prédicatif comme on vient de le voir.

81 La distinction entre proposition et syntagme sera particulièrement utile en ce qui concerne les syntagmes participiaux, parfois décrits comme des propositions ${ }^{14}$. Priscien, au VIe siècle, montre à propos du latin que la raison d'être du participe est précisément qu'il peut être adjoint aux cas obliques, autrement dit qu'il peut être incident à tout support nominal, quel qu'en soit le cas (et la fonction) :

Participium etiam oportune post verbum ponitur ... ut etiam per obliquos casus adiungi possent et sine coniunctione consociari, ut 'me legente proficio' pro 'lego et proficio'.

Le participe a été placé après le verbe ... afin que [les verbes] puissent s'adjoindre aux cas obliques et se combiner sans conjonction, par exemple 'me legente proficio' [j'avance en lisant] pour 'lego et proficio' [j'avance et je lis]. (trad. Irène Rosier)

82

Autrement dit, la raison d'être du participe est la possibilité qu'il a d'entrer, de façon plus générale, dans une relation d'incidence avec n'importe quel support nominal, indépendamment de la fonction de ce syntagme. Par ailleurs, étant incident ${ }^{15}$ à un élément nominal qui fait partie d'une structure à mode fini, le syntagme participial est ipso facto indexé sur une situation d'énoncé et partage avec l'énoncé son mode de repérage par rapport à la situation d'énonciation, ce qui fait du participe le prototype du coprédicat, dont voici un autre exemple :

23. (1. 31-32) [...] and now some artificial chill remained, quickly turning dank, carrying with it the smell of mildew.

83 L'analyse des relations prédicative et coprédicative est comparable à celle de l'exemple 22. Le prédicat remained est incident à son sujet some artificial chill, et la coprédication rend co-incidents au support some artificial chill les deux prédicats participiaux turning dank et carrying the smell of mildew.

Le syntagme participial forme avec son support une proposition à mode non fini, autrement dit un nexus. Sur le plan modal, il y a co-assertion.

L'exemple suivant contient une autre apposition, mais à mode fini :

24. (1.17-18) It slanted across the boat lots, lumberyards, and discount furniture

outlets, which already had a darkened look [...]

La relative which had a darkened look est une appositive, autrement dit une coprédication à mode fini, porteuse de temps et de modalité : elle est co-assertée (la glose and they already had a darkened look n'est qu'une approximation de sens).

\section{Subordination : intégration et enchâssement}

87 L'intégration dans un syntagme nominal, dans le cas des relatives, et l'enchâssement, dans le cas des complétives, sont compatibles avec temps, aspect et modalité (tout contenu propositionnel peut recevoir un statut modal), mais ces deux phénomènes ne sont qu'indirectement concernés par la prédication. 


\subsection{Relatives intégrées}

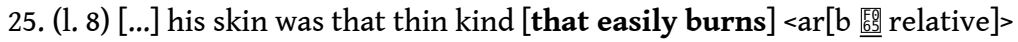

26. (1. 60) One car was slightly tipped, as if about to fall into the muddy torrent [that

churned and raced in the gully]. portant sur le contenu enchâssé (how you can see to drive). C'est la prédication de ce problème de connaissance (rhétorique, c'est-à-dire présenté comme tel sans en être un) qui motive l'énoncé, non la recherche d'une réponse, ce qui facilite la réplique de la ligne 25 ('Maybe you should put on your glasses.').

Dans l'exemple 25, that easily burns est une relative qui représente une propriété saillante de thin kind. Il s'agit d'une relative épithétique, intégrée dans le syntagme nominal au même titre que thin, mais dont la caractérisation opère sur kind déjà caractérisé par thin. Il y a un repérage de thin kind par rapport à cette propriété. Ce repérage est intégré, acquis, stable et ne donne pas de prédication. Ce qui est prédiqué, c'est la propriété de la peau du personnage (his skin was that thin kind that easily burns). La caractérisation fournie par la relative est quant à elle corrélée à la détermination that de that thin kind, qu'elle permet d'identifier. Ce qui en fait une déterminative.

Dans l'exemple 26, that churned and raced in the gully est également une relative intégrée dans le syntagme nominal, épithétique au même titre que muddy, et elle représente une propriété saillante de the muddy torrent, une caractérisation préconstruite et stable. Ce qui la distingue de la précédente (de l'exemple 25), c'est que cette propriété saillante, intégrée, stable, préconstruite, n'est pas mise à contribution pour déterminer the torrent.

\subsection{Enchâssement}

27. (1. 44) 'Well, [I don't know [that you would have]],'

28. (1.56) 'And [you don't even know [what was wrong with that]].'

29. (1. 24) '[I don't know [how you can see to drive]],'

Le schéma de l'enchâssement est dans les trois cas ar[a'r'b'], c'est-à-dire (arb tel que b= $\left.a^{\prime} r^{\prime} b^{\prime}\right)$

L'exemple 27 (1. 44) contient l'assertion d'un problème de connaissance (I don't know C1) concernant un contenu propositionnel préconstruit en amont (cf. frayage I'd have pulled over long ago), assertion qui s'interprète comme la prédication d'un doute sur le contenu enchâssé.

L'exemple 28 (1.56) est également la prédication d'un problème de connaissance. C'est l'enchâssante qui nous renseigne, et qui constitue la prédication (you don't even know C1), non le contenu enchâssé. Le statut épistémique de la subordonnée (what was wrong with that) est reçu de cet enchâssement et annoncé dans l'enchâssante. À la différence d'une interrogative directe, qui soumet un problème de connaissance au coénonciateur (ex. 1.36 'Did you notice that boy with the motorcycle?'), dans la subordonnée indirecte, l'enchâssement fait que le contenu de la subordonnée est énoncé comme problème de connaissance sans être soumis à un destinataire pour résolution.

Il en est de même dans l'exemple 29 (1. 24), assertion d'un problème de connaissance

La subordination par intégration (dans le cas des relatives) ou par enchâssement (dans le cas des complétives) laisse à l'enchâssante les caractéristiques de la prédication. 


\section{Prédication et circonstants}

30. (1. 34-35) Macon sped ahead, with his hands relaxed on the wheel. $<$ arb $>$ 熙 $\mathrm{Circ}$ 31. (1.33) [...] even before it was uttered, the hammering on the roof resumed. Circ $\underline{3}<\mathrm{arb}>$ rapport à <arb>. on the roof resumed. (2012:151-183)). ago.'

Le circonstant en sa qualité de modifieur, est dépendant. Structurellement, il est incident à une relation d'incidence. Dans l'exemple 30 (1.34-35), with his hands relaxed on the wheel est incident à <arb> (Macon sped ahead). Dans l'exemple 31, before it was uttered est incident à <arb> (the hammering on the roof resumed). Le circonstant est structurellement repéré par

En tant que modifieur, et en tant que dépendant, il est indexé sur le contenu qu'il modifie : il est circonstant de... circonstant de la représentation d'un événement ou d'un état de fait qui lui fournit l'essentiel de sa détermination énonciative. Le repérage énonciatif du circonstant s'opère par indexation. Dans l'énoncé 30, with his hands relaxed on the wheel est un circonstant dont la validité est indexée sur celle de Macon sped ahead; dans l'exemple 31, la validité de before it was uttered vaut pour the hammering on the roof resumed. Le repérage par indexation est le repérage énonciatif.

La fonction sémantico-référentielle du circonstant comme modifieur est de donner des indications sur les circonstances de <arb>. Cette fonction fait du circonstant un repère sémantico-référentiel (circonstanciel) de <arb>. Dans l'exemple 30, cette relation de repérage se représente <arb> $\underset{-65}{[60}$ Circ (repérage de arb Macon sped ahead par rapport au circonstant with his hands relaxed on the wheel); dans l'exemple 31, la relation de repérage, Circ $\underline{3}<$ arb $>$, correspond au repérage par rapport à before it was uttered, de the hammering

La prédication, comme dans les cas d'intégration et d'enchâssement observés au $§ 4$, est dans la principale, pas dans la subordonnée. En revanche, on peut se demander s'il n'y a pas coprédication dans le cas des circonstants adjoints ${ }^{16}$ (hypothèse exposée dans Merle

$30^{\prime}$ 'Macon sped ahead, and he did so with his hands relaxed on the wheel.

$31^{\prime}$ 'The hammering on the roof resumed, and this happened even before Sarah's gasp of relief was uttered.

Dans le cas des disjoints ${ }^{17}$, l'analyse est différente :

32. (1. 43) 'Sarah, if I felt we were in the slightest danger I'd have pulled over long

Le contenu de la subordonnée hypothétique (protase) If I felt we were in the slightest danger, s'interprète comme contrefactuel (I don't feel we are in the slightest danger impliqué), autrement dit comme un irréel du présent. Il sert de repère à l'apodose, c'est-à-dire à la principale I'd have pulled over long ago. Ce qui est asserté comme vraisemblable (sémantisme de would : orientation vers la validation + compatibilité sujet-prédicat), c'est le contenu irréel du passé de la principale. Mais on peut faire l'hypothèse que dans un système hypothétique, le lien entre protase et apodose est coasserté.

Dans le cas de la subordination corrélée de conséquence ([Ø] it looked as if he were only moving his lips, exemple 33, 1. 88-89), le contenu de la subordonnée est co-asserté :

33. (1. 88-89) [...] he spoke so softly, and the rain beat so loudly on the roof, [ø] it

looked as if he were only moving his lips. 


\section{Prédication sans relation prédicative?}

C'est là qu'apparait l'arbitraire de toute définition. Il y a des énoncés sans verbe, mais également sans relation <sujet-prédicat>. Dans ce cas, on peut se demander si le terme de prédication est encore applicable ou non. Si on définit la prédication comme la structuration modalisée qui fonde l'énoncé, alors le concept de prédication peut s'étendre aux énoncés sans verbes et sans relation prédicative.

34. (1.63) 'You just go on your same old way like before. Your little routines and rituals, depressing habits, day after day.'

Your little routines and rituals, depressing habits (ex. 34) est un énoncé sans verbe. On a déjà vu un assemblage prédicatif sans verbe à la ligne 58 (their windows opaque) dans lequel l'apport est le coprédicat adjectival opaque et le support le syntagme nominal their windows. Mais ici, il n'y a pas d'assemblage prédicatif. On s'aperçoit que ce segment n'est intelligible que par rapport au précédent, You just go on your same old way. Autrement dit, il s'agit d'un énoncé qui explicite le comportement (You just go on your same old way) asserté dans l'énoncé précédent. Il n'y a pas d'incidence syntaxique, mais une indexation énonciative de l'énoncé Your little routines and rituals, depressing habits, sur le précédent. Cet énoncé, en sa qualité de reformulation, a une fonction: il est porteur de modalité appréciative. Si on regarde l'énoncé suivant (exemple 35)

35. (1. 74) 'You and your systems!'

on voit qu'il s'agit également d'un énoncé modal, entièrement motivé par sa fonction appréciative. Il est également indexé sur l'énoncé précédent (I drive according to a system) sur lequel il opère un commentaire. Il s'agit d'un commentaire péjoratif, et c'est la réprobation qui motive cet énoncé. À quoi s'ajoute le surcroît d'expressivité propre à l'exclamation. Cette remarque s'applique tout aussi bien au précédent : il s'agit également d'un énoncé appréciatif, péjoratif. Ce qui est exploité dans ces deux énoncés, c'est la fonction modale propre à la prédication, le support d'indexation étant l'énoncé précédent. L'exemple 36

36. (1. 64) 'No comfort at all.'

est ambigu: il peut s'interpréter comme un prédicat de propriété attribuée au support qui précède (Your little routines and rituals, depressing habits, day after day, 1.63-64), ou comme une reprise du prédicat de la ligne 61 (discours direct 'You're not a comfort').

\section{Prédication et types d'énonciation}

\subsection{Récit}

37. (1. 2) Chips of cloudy sky showed through her tangled brown curls.

38. (1. 42) Sarah said.

Dans les deux cas, la prédication relève de la modalité de l'assertion. Le prétérit de showed et de said marque à la fois la validation de chaque relation prédicative, et une opération de repérage en rupture (c'est-à-dire une absence de point de contact entre situation d'énoncé Sit1 et situation d'énonciation primaire Sit0). La rupture est toncale dans le premier cas (constitutive d'une actualité en rupture), aoristique dans le second (renvoyant à un événement constitutif de la successivité propre au récit), événement borné à gauche et à droite dans le second cas, état de fait non borné dans le premier. 


\subsection{Discours direct}

39. (l. 61) 'You're not a comfort, Macon,' Sarah said.

La situation d'énoncé, dans l'exemple 39 (Sit1: You're not a comfort), coïncide avec la situation origine dérivée (Sitod Sarah said), elle-même en rupture (瞖) par rapport à Sit0 : Sit1 $=$ Sit origine dérivée 䁍 Sit0. La prédication est une assertion, à rattacher à la source énonciative dérivée (Sarah said), dans un énoncé de discours direct représenté (et non rapporté).

En revanche, dans l'exemple 40,

40. (1. 50-51) 'I said to you the other day, I said [Sit2 = Sit origine dérivée 2 䍖 Sit1],

'Macon, now that Ethan's dead I sometimes wonder if there's any point to life.'

on a affaire à deux situations énonciatives dérivées, et donc à du discours direct rapporté.

\subsection{Discours direct libre}

41. (1. 4) - much more logical for travelling than jeans, he always said.

112 Much more logical for travelling than jeans est du discours direct libre du premier type (sans guillemets mais avec segment introducteur), assertion de jugement sur le contenu propositionnel qui précède. De façon caractéristique, il s'agit d'un contenu de discours non extériorisé - dont l'extériorisation est sans pertinence - et récurrent, générique.

\subsection{Discours indirect}

42. (1. 51) 'Do you remember what you answered?'

113 What you answered est un contenu de discours indirect, qui se caractérise par son enchâssement, ici dans la structure d'une prédication interrogative directe. L'enchâssement de what you answered inhibe la possibilité pour ce contenu de constituer une prédication (cf. § 4.2).

\subsection{Discours indirect libre}

43. (1. 5) Jeans had those stiff, hard seams and those rivets.

Développement de l'appréciation du personnage (Macon), cet énoncé est un énoncé de discours indirect libre, c'est-à-dire non enchâssé, de second type - sans segment introducteur. On a affaire à une prédication, assertion d'une modalité appréciative dont la source est le personnage de Macon, source à laquelle on a accès par la mémoire de l'énoncé précédent.

115 La prédication est sensible aux types d'énonciation, à la double dérivation énonciative impliquée par le discours direct rapporté, à l'enchâssement caractéristique du discours indirect, qui tend à bloquer le statut de prédication des contenus indirects.

\section{Types de prédication}

\subsection{Prédication de propriété ou d'état de fait}

44. (1. 61) 'You're not a comfort, Macon,' 
116 Cette prédication de propriété met encore en jeu trois types de repérages : 1 / structurel (le prédicat be a comfort est incident au sujet you) ; 2/ sémantique (le repérage du sujet you par rapport à la propriété be a comfort est envisagé et nié) ; 3 / énonciatif, par indexation sur Sit1 élargie: la non validation est liée à la situation dérivée Sit1, mais pas exclusivement.

\subsection{Prédication d'événement}

45. (1. 84) He cleared his throat.

117 L'événement he cleared his throat est pris entre et borné par deux autres événements: Sarah said something (1. 82) et He said, 'Honey.' (1. 84). L'interprétation aoristique du prétérit est activée.

\subsection{Prédication d'existence}

46. (1.15-16) [...] there was a gentle patter on the roof.

118 There, support structurel de prédication, marque une opération de localisation. À lui seul, cependant, il ne suffit pas à représenter la situation. La situation est représentée de façon solidaire par there et par be muni de sa détermination (donc there + [temps + be]). Le repérage mis en place est celui de a gentle patter par rapport à une situation en rupture (rupture marquée par le prétérit, rupture entre situation d'énonciation et situation d'énoncé) et la localisation spatiale est fournie par le troisième élément on the roof; l'existence en soi implique une localisation. Mais ce troisième élément peut ne pas apparaître: la localisation s'opère alors simplement par rapport à la situation, représentée par [there + (temps + be)], there was dans l'exemple 47 (1. 13) :

47. (l. 13) There wasn't much traffic.

\subsection{Prédication de jugement}

L'exemple type est la structure à extraposition, comme à la ligne 40 :

48. (1. 40) 'It's crazy to ride a motorcycle on a day like today,'

Ici, on a affaire à une prédication - it's crazy - de jugement, appréciatif, asserté, sur le contenu propositionnel extraposé (to ride a motorcycle on a day like today). Ce jugement est indexé sur la situation mais sa validité n'est pas limitée à la situation (repérage situationnel élargi). On retrouve l'incidence structurelle, mais pas de repérage sémantique : il ne s'agit pas d'attribuer une propriété au sujet it, qui n'est en fait que support syntaxique (cf. $\$ 1.3 .6$ ), mais d'asserter le jugement. Remarque : la modalité est appréciative, mais elle est souvent épistémique dans ce genre de structure. L'exemple 49 est un autre cas de prédication de jugement :

49. (1. 74) 'You and your systems!'

\section{9. Étude de cas et 'carré des modaux' (Adamczewzki et Gabilan)}

121 Dans les exemples sélectionnés (50 à 53), on a systématiquement affaire à des prédications verbales et porteuses de temps. On rappellera que la fonction verbale est nodale, modale, prédicative. Et que c'est toujours le premier élément verbal qui noue la 
relation prédicative. L'inversion auxiliaire-sujet, comme à la ligne 19, dans un énoncé interrogatif direct, s'opère ainsi toujours avec le premier auxiliaire, puisque la ligature prédicative est remise en cause selon le sémantisme du premier élément verbal, quatre sous-paradigmes étant activables (be, have, do, modaux).

\subsection{Do et l'enjeu de la validation / non-validation de la relation prédicative}

50. (1. 6-7) Sarah had a tan but Macon didn't.

51. (1. 48) 'I don't know that you really care that much,' Sarah said. 'Do you.'

52. (1. 65-67) 'I don't know why you feel it's your loss alone.'

'Well, I just do, sometimes.'

53. (1. 90) 'You can keep the house,' Sarah said. 'You never did like moving.'

Ce qui est en cause, ici, avec do, c'est la gestion de la polarité, autrement dit la validation ou la non-validation de la relation prédicative : 1.6 entre Macon et have a tan; 1.48 , entre you et care - c'est ainsi que le comprend Macon : cf. 1. 49, 'Care?'; 1. 67, entre I et feel it's my loss alone ; 1.90 , entre you et like moving.

Dans les trois premiers cas $(50,51,52)$, le prédicat concerné a déjà été mentionné. Et il n'est pas explicité dans la prédication envisagée. Dans le quatrième cas (53), il n'y a pas de mention préalable.

Mais dans tous les cas, il y a frayage, dans les trois premiers exemples par la première mention, dans la troisième situation parce qu'il s'agit d'une scène intime, entre les deux époux, et que la locutrice connaît le colocuteur de façon intime.

À la ligne 6 (50), le même prédicat (had a tan) est appliqué d'abord au sujet S1 Sarah, selon une relation validée, puis ré-envisagé par rapport au sujet S2 Macon. La masse lexicale du prédicat étant la même pour les deux supports, l'ellipse permet non pas de faire des économies mais de construire un lien anaphorique entre les deux relations. Le contenu propositionnel est validé dans un cas, non validé dans l'autre: $\mathrm{p}$ pour un sujet, $\mathrm{p}$ ' pour l'autre. La non validation pour S2 est marquée au niveau de l'élément qui remplit la fonction nodale et modale, et on a donc didn't.

À la ligne 48 (51), pour un même sujet you, on a affaire à la remise en cause du contenu propositionnel you care much. La prédication est assertion d'un doute (I don't know) sur la validité du contenu enchâssé that you care that much; l'anaphore est manifeste: that1 subordonnant (sans possibilité de subordonnant $\varnothing$ ) ; that2 adverbial ; really, rappel de la remise en doute; $\mathrm{p}$ (validation de <you-care much>) est à mettre sur le compte du colocuteur ; p', non validation de <you-care much> est rendu pertinent par la mise en doute; ce qui est prédiqué dans le tag, c'est la position contraire, p. La fonction pragmatique de ce tag s'accompagne comme dans le cas précédent (50, ligne 6) d'un phénomène d'anaphore construit sur l'ellipse caractéristique du tag.

À la ligne 67 (52), on a un centrage sur do, marqueur de validation de <S-feel it's S's loss alone>, avec ellipse du prédicat, comme dans les deux cas précédents, ellipse dont le rôle n'est toujours pas de faire des économies, mais d'activer le fonctionnement mémoriel qui caractérise l'anaphore. Ce centrage est opéré par le focalisateur (l'adverbe paradigmatisant) just. Glose : c'est comme ça (focalisation sur do, et donc sur la validation de la relation prédicative) et pas autrement (prise en compte d'un paradigme). Le paradigme pris en compte est celui ouvert par why dans l'énoncé précédent (why you feel it's your loss alone) ; glose : c'est comme ça, inutile de chercher pourquoi. 
À la ligne 90 (53), la négation porte sur le lien prédicatif. La prédication est une assertion. La non-validation p' du contenu propositionnel correspondant à la relation prédicative < you-like moving> est privilégiée, et $\mathrm{p}$ (validation de ce contenu) est explicitement écarté.

\subsection{Would : orientation vers la validation et compatibilité sujet- prédicat}

54. (1. 29) [...] if you looked closely you'd notice the tension at the corner of her eyes.

On remarquera d'abord qu'il existe virtuellement deux interprétations possibles de la protase - comme subordonnée de condition (adjointe) et comme subordonnée hypothétique (disjointe) - mais une seule interprétation de l'apodose et du lien de solidarité entre protase et apodose.

La première interprétation, celle de la protase comme subordonnée de condition, permettrait à la subordonnée d'entrer sous la portée d'un focalisateur (propriété des circonstants adjoints): It was only if you looked closely that you'd notice the tension at the corner of her eyes. / It took a close look to notice the tension. L'interprétation serait alors que la compatibilité entre le sujet you et le prédicat notice the tension at the corner of her eyes dépendrait étroitement de la condition if you looked closely, autrement dit de la validation de la relation <you-look closely>, ce qui reviendrait à dire que la tension est perceptible, mais perceptible seulement pour un œil attentif. Un élément du cotexte vient invalider cette interprétation : but (1.29).

La seconde interprétation de la protase comme disjoint hypothétique est donc seule acceptable. La glose de l'hypothèse serait alors if you happened to take a close look, you'd notice the tension at the corner of her eyes. Le système hypothétique rend solidaires la validation de la protase (if you looked closely) et l'orientation vers la validation du contenu de l'apodose (you'd notice the tension at the corner of her eyes).

Dans l'apodose, would exprime donc une orientation vers la validation de syou-notice the tension at the corner of her eyes (validation prévisible) et la compatibilité entre le sujet you et le prédicat notice the tension at the corner of her eyes en fonction de l'hypothèse (solidarité protase-apodose).

\subsection{Suggestion de révision du carré des modaux d'Adamczewzki \& Gabilan ${ }^{18}$}

133 Là où Adamczewzki \& Gabilan utilisent le terme d'orientation vers la prédication pour renvoyer à l'idée d'orientation vers la validation de la relation qui se noue autour des modaux, je substitue orientation vers la validation pour rétablir une distinction entre validation et prédication selon les définitions données au début de cet article, la prédication étant définie comme structuration compatible avec toutes les modalités et avec tous les statuts illocutoires, et la plurimodalité étant une caractéristique courante de la prédication.

Dans la colonne de gauche, j'ajoute une opposition: dépendance interne VS dépendance externe.

La dépendance interne s'interprète comme "interne à l'énoncé » et correspond aux modalités radicales dynamiques, concernée par la compatibilité a priori entre sujet et 
prédicat. La dépendance externe s'interprète comme « externe à l'énoncé » et correspond aux modalités radicales déontiques, qui expriment une intervention de l'extérieur accordant à $\mathrm{S}$ (permission) ou imposant à $\mathrm{S}$ (contrainte) un prédicat $\mathrm{P}$, ce qui implique une absence de compatibilité a priori.

A priori est un autre ajout: la compatibilité ou l'absence de compatibilité ne sont pas à comprendre de façon absolue, mais sont incluses a priori dans le sémantisme et dans la pertinence de la modalité dans le contexte propre à chaque prédication : a priori permet d'intégrer l'éventualité du mensonge ou de l'ironie, mais également d'anticiper sur un contexte polémique. La (pluri)modalité propre à la prédication est une prise de position propre à une situation d'énonciation donnée.

\begin{tabular}{|l|l|l|}
\hline & $\begin{array}{l}\text { +Orientation vers } \\
\text { validation de la RP }\end{array}$ & $\begin{array}{l}\text {-Pas d'orientation vers } \\
\text { validation de la RP }\end{array}$ \\
\hline $\begin{array}{l}\text { S-P a priori } \\
\text { Dépendance interne à l'énoncé }\end{array}$ & WILL / WOULD & CAN / COULD \\
\hline $\begin{array}{l}\text { - Pas de Compatibilité / - Pas de } \\
\text { Congruence S-P a priori } \\
\text { Dépendance externe à l'énoncé }\end{array}$ & SHALL / SHOULD & MAY / MIGHT \\
\hline
\end{tabular}

\section{Conclusion}

Ce tour d'horizon a permis de définir la prédication comme opération énonciative structurante et modalisée, et de revenir sur différentes approches, parmi lesquelles l'approche néoplatonicienne (prédicat central) et l'approche néo-aristotélicienne (apport prédicatif sur substrat) sont les plus convaincantes. On a vu que la modalité énonciative associée à la prédication est dans les faits très souvent plurimodalité, ce qui en fait un domaine à explorer. On a comparé deux phénomènes connexes, la biprédication, dans un contexte de coordination, qui peut s'assortir de modalités énonciatives autonomes, et la coprédication, dont la dépendance étroite (fonction prototypique, l'apposition; constituant prototypique, le participe) tend à favoriser sur le plan modal la co-assertion. On a observé que les subordonnées relatives intégrées et les subordonnées nominales (enchâssées) ne donnent pas lieu à prédication et sont dépendantes d'une prédication qui se trouve dans la structure d'accueil. On a observé le lien entre prédication et circonstants et on a fait l'hypothèse que le lien entre prédication et circonstants pouvait être d'ordre coprédicatif. On a remarqué que la définition de la prédication comme structuration modalisée qui fonde l'énoncé était compatible avec des énoncés sans verbe et sans relation prédicative. On a observé à plusieurs reprises les manifestations des trois repérages qui structurent les énoncés : repérage structurel, par incidence; repérage énonciatif, par indexation; repérage sémantico-référentiel, qui tend à fonctionner à rebours des deux précédents. Les études de cas sont centrées sur do, concerné par l'opposition entre validation et non-validation, et sur would, qui exprime une orientation 
vers la validation et une compatibilité sujet-prédicat. Cette dernière étude a été l'occasion de revenir sur le carré des modaux d'Adamczewzki et Gabilan.

\section{BIBLIOGRAPHIE}

Adamczewzki H. et Gabilan J.-P. (1993). Les clés de la grammaire anglaise, Paris : Armand Colin. Albrespit J. (2003). Sujet et agentivité en anglais, in Le sujet (dir. J.-M. Merle), Bibliothèque de Faits de Langues, Paris / Gap : Ophrys, 125-136.

Cotte P. (1984). « Remarques sur les incidences dans la phrase nucléaire du français et de l'anglais », in Modèles linguistiques, tome VI, 2, Lille : Presses Universitaires de Lille, 129-140.

Creissels D. (1995). Eléments de syntaxe générale, Paris : Presses Universitaires de France.

Culioli A. (1990). Pour une linguistique de l'énonciation - tome 1 : Opérations et représentations, Paris : Ophrys.

Culioli A. (1999a). Pour une linguistique de l'énonciation - tome 2 : Formalisation et opérations de repérage, Paris : Ophrys.

Culioli A. (1999b). Pour une linguistique de l'énonciation - tome 3 : Domaine notionnel, Paris : Ophrys.

Danon-Boileau L. (1987). Énonciation et référence. Paris / Gap : Ophrys.

Ildefonse F. (1994). « Sujet et prédicat chez Platon, Aristote et les Stoïciens », in Archives et documents de la société d'histoire et d'épistémologie des sciences du langage, J. Lallot (dir.), $\mathrm{n}$ • 10, Université Paris 7.

Lallot J. (1994) (dir.) Archives et documents de la société d'histoire et d'épistémologie des sciences du langage, seconde série $n^{\circ} 10$, décembre 1994, Paris 7 et SHESL.

Lazard G. (2003). Le sujet en perspective interlinguistique, in Le sujet, J.-M. Merle, (dir.) Bibliothèque de Faits de Langues, Paris / Gap : Ophrys, 15-28.

Merle J.-M. (2000). The French conditional vs WOULD + Verb (communication à Berkeley), http:// hal.archives-ouvertes.fr/hal-00670010

Merle J.-M. (2001). Étude du conditionnel français et de ses traductions en anglais, Paris / Gap : Ophrys.

Merle J.-M. (2003). Présentation générale du volume Le sujet, J.-M. Merle (dir.), http:// hal.archives-ouvertes.fr/hal-00378857, in Bibliothèque de Faits de Langues, Paris / Gap : Ophrys, $5-14$.

Merle J.-M. (2006a). «Wh- et la référence qualitative aux paradigmes », in Le Qualitatif, J.C. Souesme (dir.), Cycnos, vol. 23, n 1, Université de Nice, 25-43.

Merle J.-M. (2006b). Structures et références. https://tel.archives-ouvertes.fr/tel-00672594.

Merle J.-M. (2008). « Prépositions et aspect », L'Information grammaticale, $n^{\circ} 117$, D. Leeman (dir.) Louvain / Paris : Peeters, 52-56. http://hal.archives-ouvertes.fr/hal-00433875/fr/ 
Merle J.-M. (2009a). Présentation générale de La prédication, collection Faits de Langues, Paris : Ophrys, 5-12. http://hal.archives-ouvertes.fr/hal-00374896.

Merle J.-M. (2009b). Les constructions participiales en anglais, in La Prédication, J.-M. Merle (dir.), Faits de langues, $\mathrm{n}^{\circ}$ 31-32, ISBN 978-2-7080-1224-0, Paris : Ophrys. 2009, p. 413-422, http:// hal.archives-ouvertes.fr/hal-00433874

Merle J.-M. (2012). « La qualification, phénomène protéiforme - à propos de quelques manifestations syntaxiques de la qualification en anglais et en français », in La qualification, J.M. Merle (dir.), Travaux du CLAIX n 22, Aix-en-Provence : Presses universitaires de Provence, 151-184 : http://hal.archives-ouvertes.fr/hal-00671423

Neveu F. (2000). «L'apposition : concepts, niveaux, domaines », in Nouvelles recherches sur l'apposition, Langue française $\mathrm{n}^{\circ} 125$, Paris : Larousse.

Quirk R., Greenbaum S., Leech G., Startvik J., Crystal D. (1985). A Comprehensive Grammar of the English Language, Londres, New York : Longman.

Touratier C. (2003). « Sujet et syntaxe ?», in Le sujet, J.-M. Merle (dir.), Bibliothèque de Faits de Langues, Paris / Gap : Ophrys, p. 41-50.

Vincent-Durroux L . (2010). « La dislocation en anglais : phénomène(s) syntaxique(s) et motivations sémantiques ». Les amis du CRELINGUA, $n^{\circ}$ 15, Paris : Editions EMA. p. 52-61.

Wilmet M. (1997a). Grammaire critique du français. Paris : Duculot, Hachette Supérieur.

Wilmet M. (1997b). « L'apposition : une fonction à réestimer », in Les formes du sens. Etudes de linguistique française, médiévales et générales offertes à Robert Martin, G. Kleiber et M. Riegel (dir.), Louvain-La-Neuve : Duculot, 413-422.

\section{NOTES}

1. La définition et une partie de la présentation des trois approches de la prédication ont été empruntées à la présentation générale de Merle (2009a).

2. Trad. M. Baratin et F. Desbordes, apud F. Ildefonse (1994), p. 6

3. Ibidem

4. Catégories, 2, 1a20-1b6, trad. J. Tricot, apud Ildefonse (1994), p. 11

5. Métaphysique, 2, 1, 1028a 10-20, trad. J. Tricot, ibidem, p. 12

6. III $^{\mathrm{e}}$ siècle de notre ère. Source : Vies et opinions des philosophes antiques, VII, 63, trad. F. Ildefonse, ibidem, p. 25

7. Trois unités lexicales.

8. La catégorie du pronom se définit par sa fonction syntaxique nominale (et non par une éventuelle fonction anaphorique : tous les pronoms n'ont pas d'antécédent) : le pronom est la catégorie grammaticale qui correspond à la catégorie lexicale du nom.

9. De façon anecdotique, la notion d'apposition désigne, chez certains linguistes (cf. Quirk \& alii 1985), une épithète nominale dans une relation de coréférence avec le noyau du syntagme nominal, sur le modèle urbs Roma (la ville de Rome = Rome). Cf. Neveu (2000), pour une étude complète de la problématique de l'apposition.

10. À propos de la problématique du sujet vu comme substrat, cf. Ildefonse (1994); sur la bipartition entre sujet de prédication et sujet de référence, et sur le couple sujet-prédicat envisagé comme une grammaticalisation du couple thème-rhème, cf. Lazard (2003) ; sur la complémentarité entre fonction subjectale (sujet de prédication comme terme de départ) et fonction référentielle du sujet ou d'un élément disloqué (repère constitutif de l'énoncé dans la TOE), cf. Vincent- 
Durroux (2010) ; sur la distinction entre sujet, thème et agent, cf. Albrespit (2003) et Touratier (2003) ; cf. également Merle (2003) sur les problèmes que pose la définition du sujet.

11. Incidence : relation syntaxique ordonnée, correspondant à un repérage structurel ; ainsi her head contient une relation d'incidence de her déterminant, à head, déterminé.

12. La glose donne une approximation de sens.

13. Les appositions sont des prédications.

14. C'est ainsi que nous en arrivons à nier l'existence du syntagme participial, de trois façons différentes: dans la structure des constituants, en le décrivant comme une proposition participiale, puis en nommant cette " proposition participiale " « relative réduite "; et dans la structure fonctionnelle, en lui donnant la fonction de circonstant, alors que le syntagme participial est toujours incident à un support nominal. Dans Frowning, I looked at my watch, (A. Garland), frowning est un syntagme participial apposé co-incident avec looked at my watch à son support I: frowning est co-prédicat incident à un support nominal. La relation d'incidence entraîne une indexation du nexus (formé par la mise en relation du co-prédicat frowning et de son support I) sur I looked at my watch, et donc une relation étroite entre le nexus et la proposition. Cette relation s'interprète ici comme une relation de concomitance simple (sans relation de cause à effet ni d'opposition concessive).

15. Rappel: la relation d'incidence se définit comme une mise en relation syntaxique (structurelle) d'un apport et d'un support.

16. Les circonstants adjoints représentent l'environnement sémantico-référentiel des événements et des états de fait. Ils réagissent pour cette raison aux tests de focalisation (it was [not] before Sarah's gasp of relief was uttered that the hammering on the roof resumed, [but immediately afterwards]).

17. Les disjoints, "circonstants de l'énonciation », concessifs, hypothétiques, ne correspondent pas aux circonstances naturelles des événements et des états de fait, mais à l'arbitraire énonciatif. Pour cette raison, ils ne réagissent pas aux tests de focalisation.

18. Adamczewzki et Gabilan (1993).

\section{RÉSUMÉS}

La prédication est définie ici comme opération énonciative structurante ( $\$ 1$ ) à laquelle sont associées les modalités énonciatives. Trois approches différentes de la prédication sont examinées dans cet article. On envisage la question de la modalité énonciative et celle de la plurimodalité (\$2). On observe ensuite ( $\$ 3$ ) deux phénomènes connexes : la biprédication, dans un contexte de coordination; et la coprédication. On s'interroge sur les subordonnées relatives enchâssées - adjectivales comme nominales - et sur les raisons pour lesquelles elles ne donnent pas lieu à prédication $(\$ 4)$. On envisage le lien entre prédication et circonstants $(\$ 5)$, puis un cas particulier ( $\$ 6$ ), la prédication sans relation prédicative. On amorce deux typologies : celle du lien entre prédication et types de discours $(\S 7)$; puis celle des types de prédication $(\S 8)$. La dernière partie (§ 9) est consacrée à cinq études de cas.

Predication is defined here as an enunciative operation structuring utterances (§ 1), along with enunciative modalities. The issue of illocutionary status is addressed here in the framework of plurimodality $(\$ 2)$. Three different approaches of predication are presented in this paper. Then two peculiar types of predication ( $(3)$ are examined : bipredication, in a context of coordination ; 
and copredication, occurring with predicative adjuncts. Embedded (integrated) relative clauses whether adjectival or nominal - are not predications ( $\$ 4)$. The link between predication and adverbials is examined in $\S 5$, and then in $\S 6$, the case of predication with no predicative relation. Two typologies are sketched out in the next two sections : predication and types of discourse in $\S 7$; types of predication in $\S 8$. The last section ( $(9)$ is centred on five case studies.

INDEX

Mots-clés : prédication, coprédication, biprédication, plurimodalité, relatives, modalité, circonstants, types de discours

Keywords : predication, copredication, bipredication, plurimodality, relative clauses, modality, adverbials, types of discourse

\section{AUTEUR}

\section{JEAN-MARIE MERLE}

Université Nice Sophia Antipolis

BCL (Bases, corpus, langage) (UMR 7320)

jean-marie.merle@unice.fr 